\title{
Tumores neuroendocrinos primarios de colon y recto
}

\author{
Guillermo Bannura ${ }^{1}$, Alejandro Barrera ${ }^{1}$, Carlos Melo $^{1}$, \\ Felipe Illanes ${ }^{1}$ y Cristian Gallardo ${ }^{1}$
}

\section{Primary neuroendocrine tumors of the colon and rectum}

Background: The nomenclature and staging classification of neuroendocrine tumors (NETs) has changed drastically in the past decade. Objective: To do a critical analysis of management of colorectal NETs in our institution in the light of the new classification. Methods: We retrospectively reviewed the records of consecutive patients operated on with radical intention due to a colorectal NET in the last 15 years. Results: There were 10 patients, median age was 56 years (range 48-76), six of them located near the ileocecal valve, three in the rectum ( 2 of them polyps) and one polyp in the sigmoid colon. Surgical procedure included four anterior resections and six right colectomy (one with hepatic resection). The median follow up was 78.3 months (range 8-180). Two patients died due to metastatic disease. Conclusion: NETs located near de ileocecal valve were diagnosed usually as a big tumor with obstructing symptoms, while NETs of the rectum and sigmoid colon more frequently were detected as polyps or submucosal lesions. Some low grade TENs may invade the colonic wall and/or have metastasis in the regional lymph nodes and those cases need radical resection and/or adjuvant therapy. Combine the grade (Ki-67 and number and/ or number of mitosis) of $2010 \mathrm{WHO}$ classification with TNM showed prognostic value for classification and staging colorectal NETs with important therapeutic implications.

Key words: Neuroendocrine tumors; Colorectal; Surgery.

\section{Resumen}

Introducción: La clasificación y el manejo de los tumores neuroendocrinos (TNEs) han cambiado drásticamente en la última década. Se realiza un análisis crítico del manejo de los TENs colorrectales primarios intervenidos en nuestro centro a la luz de la nueva clasificación. Material y Método: Análisis retrospectivo de los resultados del tratamiento quirúrgico radical de este tipo de lesiones en un período de 15 años. Resultados: Se trata de 10 pacientes, con un promedio de 56 años (extremos 48-76), 6 ubicados cercanos a la válvula ileocecal (VIC) que se presentaron con un cuadro de obstrucción intestinal incompleto y tres en el recto (2 pólipos) y un pólipo en colon sigmoides. La cirugía efectuada incluye cuatro resecciones anteriores y seis colectomías derechas (con resección de una metástasis hepática en 1 caso). La mediana de seguimiento fue de 78,3 meses (extremos 8-180), durante el cual dos pacientes fallecen por metástasis a distancia. Conclusión: Los TNEs ubicados en el colon habitualmente se presentaron como grandes masas tumorales cercanos a la VIC, lo que proporcionalmente no ocurre con las lesiones del rectosigmoides que son diagnosticados más precozmente como pólipos o lesiones submucosas. Algunos tumores de bajo grado muestran un compromiso avanzado en la pared y/o los linfonodos regionales lo que justifica la resección radical y/o la quimioterapia adyuvante. A la luz de esta experiencia, es necesario complementar el grado del tumor OMS 2010 (Ki-67 y número de mitosis) con el estadio TNM para caracterizar adecuadamente los TNEs, lo que influye en el manejo multidisciplinario.

Palabras clave: Tumores neuroendocrinos; Cirugía; Carcinoides colon y recto.
Servicio y Departamento de Cirugía, Hospital Clínico San Borja Arriarán, Campus Centro, Facultad de Medicina Universidad de Chile. Santiago, Chile.

Recibido el 3 de abril de 2017 aceptado para publicación el 28 de junio de 2017.

Correspondencia a: Guillermo Bannura gbannura@gtdmail.com 


\section{Introducción}

Los tumores neuroendocrinos (TNEs, en inglés NETs) son tumores raros que constituyen alrededor de 1 a $2 \%$ de todas las neoplasias ${ }^{1}$. El aparato digestivo y el páncreas son el asiento del $65 \%$ de los TNEs, seguido del sistema broncopulmonar (25\%), mientras que el $10 \%$ restante se localiza en el sistema urogenital, cabeza y cuello, mama y piel $^{1,2}$. La localización más frecuente en el tracto digestivo es el apéndice, el íleon y el recto, con notables diferencias en el comportamiento biológico según el segmento afectado ${ }^{1-3}$.

El estudio, la nomenclatura y el tratamiento de los TNEs ha experimentado numerosas modificaciones en la última década. La clasificación de la OMS de 2002 de los TNEs se basó en el grado de diferenciación tumoral y el tamaño de la lesión, lo que fue reemplazado en la clasificación del año 2010 por el índice de proliferación celular (Ki-67) y/o el número de mitosis por 10 campos de aumento mayor $(\mathrm{IM} \times 10 \mathrm{CAM})^{4}$. El grado del tumor muestra una correlación muy marcada con el pronóstico de estas lesiones (Tabla 1). La Sociedad Europea de Tumores Neuroendocrinos (ENETS) ${ }^{5}$ desde 2006 utiliza el sistema TNM/AJCC para la estadificación de este tipo de lesiones, la que ha sido validado en varios estudios y aceptada por la Sociedad Americana de Tumores Neuroendocrinos (NANETS) ${ }^{6}$. Aunque el término carcinoide aún lo utilizan algunos autores para designar a los TNEs de bajo potencial maligno (G1), la tendencia es emplear la nueva clasificación de la OMS según el grado de la lesión complementada con la estadificación TNM (Tabla 2).

En nuestro medio, hemos encontrado una publicación del año 2003 sobre un caso de un TNE del anorrecto ${ }^{7}$, lo que ha motivado una revisión crítica del manejo de los NETs colorrectales en nuestro centro y proponer un tipo de estudio y manejo terapéutico de estas lesiones, a la luz de la nueva nomenclatura y clasificación.

\section{Material y Método}

Se trata de una revisión retrospectiva de los TNEs colorrectales primarios tratados en un período de 15 años (septiembre de 2001-agosto de 2016), información obtenida de una base de datos recolectada en forma prospectiva. Se incluyen los pacientes en los cuales el estudio histopatológico y/o inmunohistoquímico (IHQ) confirmó un tumor NE y que fueron sometidos a una resección radical del segmento comprometido. Se excluyen los carcinoides del apéndice cecal y los pólipos rectales resecados por vía endoscópica o sometidos a una resección local. Se analizan los antecedentes clínicos, el estudio preoperatorio, el tipo de cirugía efectuada, la morbilidad postoperatoria y el seguimiento a largo plazo. Se revisa en detalle el estudio histopatológico de los casos, registrando cuando el dato existía, los estudios de inmunohistoquímicas y la medición de los IM y Ki-67 efectuados. Se empleó la clasificación histopatológica de la OMS de $2010^{4}$ y el TNM para la estadificación de los $\operatorname{casos}^{5,6}$.

\section{Resultados}

En el período señalado se intervinieron 10 pacientes por un TNE colorrectal, 6 del género masculino, con un promedio etario de 56 años (extremos 48-76) (Tabla 3). Los síntomas más comunes fueron la baja de peso en 6 casos, diarrea crónica en 5, masa abdominal en 4 y dolor abdominal tipo cólico en 3. Los seis casos ubicados cercanos a la válvula ileocecal se presentaron con un cuadro de obstrucción intestinal incompleto. Un paciente de 53 años (caso 3) se hospitalizó de urgencia debido a deshidratación y alteración electrolítica secundaria a una diarrea profusa. La colonoscopía mostró un gran tumor protruido de $15 \mathrm{~cm}$ de consistencia blanda ubicado en el recto superior. Este estudio mostró inicialmente en 3 pacientes un pólipo (casos 1,9 y 10), mientras que en los 6 pacientes restantes se encontró una lesión tumoral de grandes dimensiones a nivel de la región cecal. La TC fue normal en 3 pacientes portadoras de un pólipo e informó de un tumor avanzado en el resto, dos de ellos con metástasis hepáticas (casos 7 y 9). En los casos 7 y 10 se efectuó un PET-CT con 68Ga-Dotatate que mostró un foco de sobreexpresión de los receptores de somatostatina en la región ileocecal y en la unión rectosigmoidea, respectivamente.

La cirugía efectuada incluye 4 resecciones anteriores y seis colectomías derechas (con resección de una metástasis hepática en 1 caso). La evolución postoperatoria fue satisfactoria en 9 casos con un promedio de hospitalización de 5 días. El caso 7 fue reoperado al $6^{\circ}$ día por una dehiscencia de sutura, falleciendo a los 32 días por sepsis y falla orgánica multisistémica. En el caso n ${ }^{\circ} 1$, resecado inicialmente por vía endoscópica, el estudio histopatológico del pólipo reveló un tumor NE bien diferenciado con margen profundo positivo, motivo por el cual se programó para una resección anterior. La biopsia de la pieza resecada no reveló tumor y los linfonodos fueron negativos para metástasis. El detalle de estu- 
dio histopatológico se resume en la Tabla 4 (Figuras 1 y 2 : H\&E e IHQ caso $n^{\circ} 9$ ). El seguimiento tiene una mediana de 70,5 meses (extremos 8-180). Dos pacientes (casos 2 y 3) sin tratamiento sistémico fallecen por metástasis hepáticas múltiples a los 13 y 96 meses, respectivamente. La correlación entre el grado de la lesión según la OMS, el estadio TNM y la sobrevida se muestra en la Tabla 5.

\section{Discusión}

Los TNEs colorrectales constituyen un grupo heterogéneo de tumores con diferenciación neuroendocrina, representa el 1 al 4\% del total de las neoplasias de esta localización, predomina entre la sexta y séptima década de la vida y la localización más frecuente es el ciego, el recto y el sigmoides ${ }^{1-3,8,9}$, lo que se aprecia en esta pequeña serie que no incluye los pólipos rectales extirpados o sometidos a resección local. En el pasado se emplearon diferentes clasificaciones y nomenclaturas de acuerdo a las características histopatológicas, lo que claramente condiciona el tratamiento de estas lesiones. Los estudios comparativos de diferentes series confirman la variabilidad de los tratamientos efectuados a pacientes con estadios similares ${ }^{10}$.

Las guías clínicas de consenso entre la OMS, ENETS, NANETS y AJCC destacan la importancia de combinar el estadio TNM con el grado tumoral según el índice mitótico y el índice Ki-67, los cuales se consideran actualmente como los elementos pronósticos más relevantes ${ }^{9-12}$. Del análisis de nuestros casos se confirma la importancia de estadificar según el TNM, toda vez que 3 de 4 pacientes con un tumor grado 1 correspondían a un estadio IIIA (T4), IIB (T3) y IIIB (compromiso de los linfonodos), respectivamente, lo que justificó una resección radical a pesar del bajo grado. El caso $\mathrm{n}^{\circ} 7$, a pesar de ser grado 1, tenía al momento de la cirugía linfonodos metastásicos y metástasis hepática (estadio IV). Asimismo, lesiones grado 2 (intermedio), recibieron quimioterapia adyuvante debido al compromiso metastásico de los linfonodos regionales (casos 8 y 9).

En un estudio multicéntrico realizado en Japón sobre 760 NETs colorrectales tratados ${ }^{9}, 233$ pacientes fueron sometidos a una resección radical por una lesión del recto. Los factores predictivos

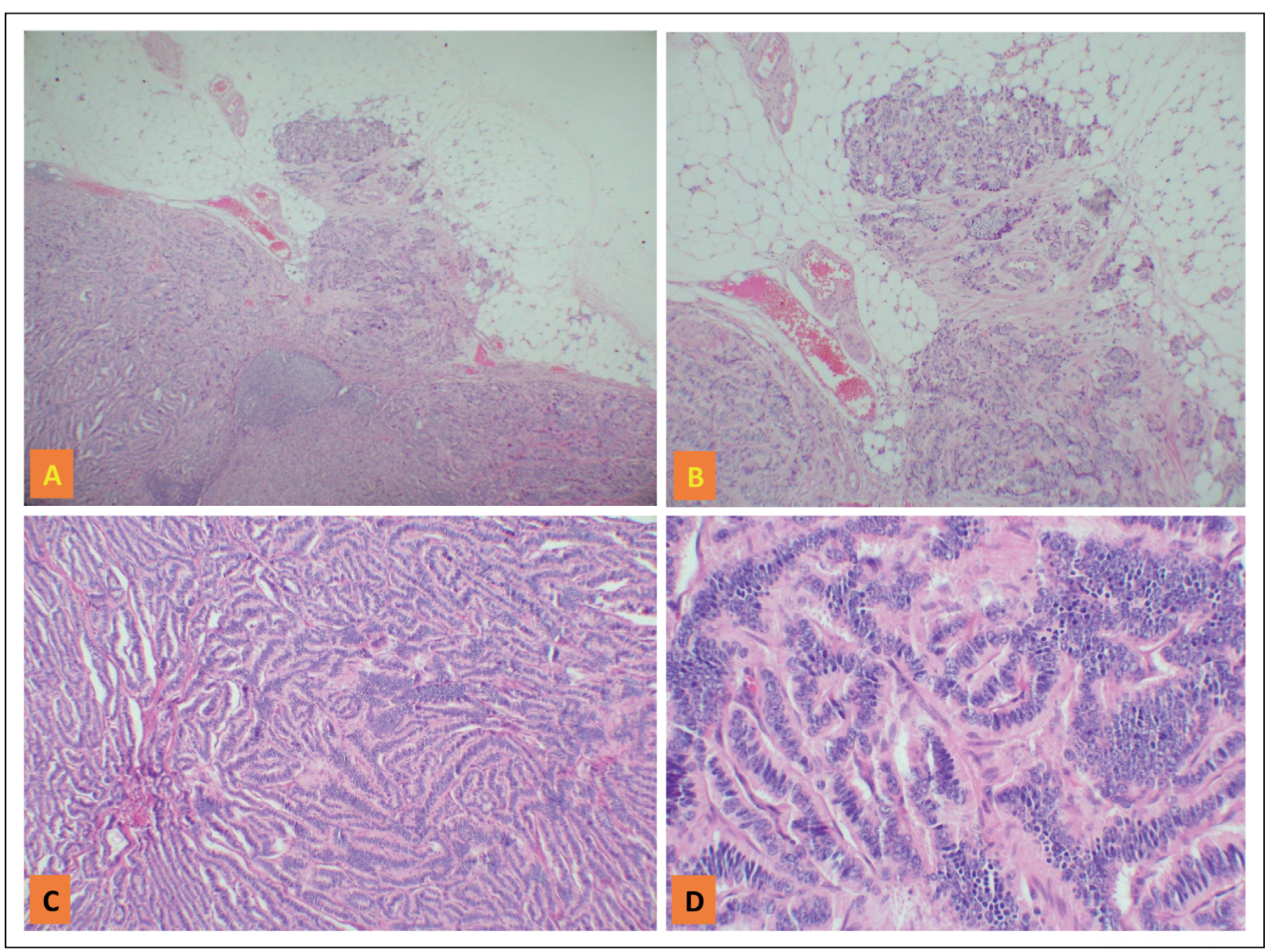

Figura 1. Tinción con hematoxilina-Eosina (HE). A y B. $40 \times \mathrm{HE}$ y $100 \times \mathrm{HE}$, metástasis ganglionar con extensión extracapsular. C y D. $100 \times \mathrm{HE}$ y $400 \times \mathrm{HE}$, metástasis ganglionar. 


\section{ARTíCULO DE INVESTIGACIÓN}

Figura 2. Inmunohistoquímica (IHQ). A. Sinaptofisina, positiva difusa patrón citoplasma. B. 400 $\mathrm{x}$, Cromogranina, positiva focal patrón citoplasma. C. $100 \mathrm{x}$, índice de proliferación Ki67, positivo patrón nuclear. D. $400 x$, Índice de proliferación Ki67, positivo pa.

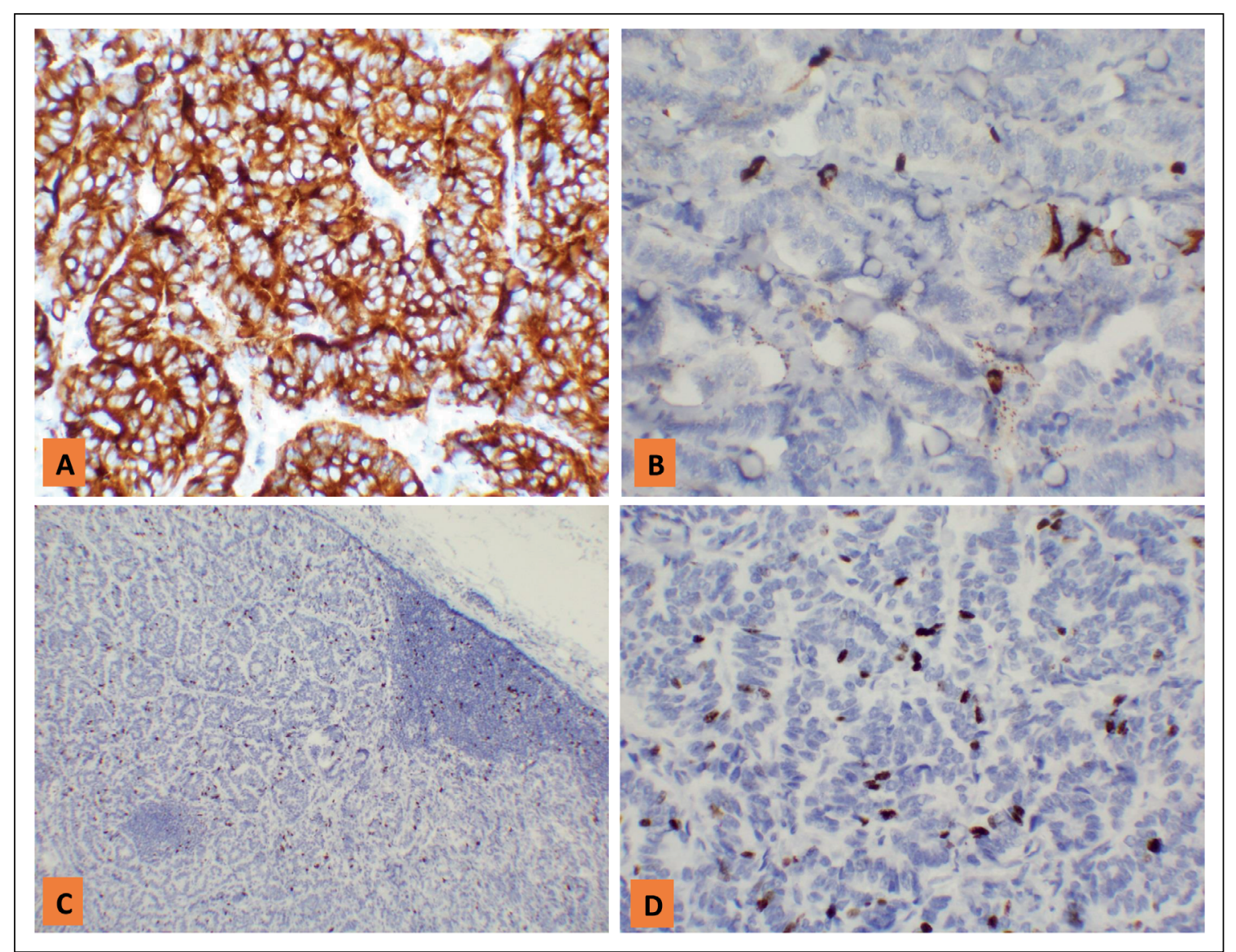

del compromiso de los ganglios linfáticos en el análisis multivariado fueron el tamaño de la lesión, la profundidad, el índice mitótico, el índice Ki-67, la invasión linfática y vascular. Notablemente, el riesgo de metástasis ganglionar en lesiones menores de $10 \mathrm{~mm}(\mathrm{n}=98)$ fue 9,2\%. En una revisión sistemática que incluyó 1.225 pacientes con un NETS de colon y recto, de los cuales 515 fueron sometidos a una resección radical ${ }^{13}$, se destaca como factores predictivos de compromiso linfonodal el tamaño de la lesión (> $1 \mathrm{~cm}$; OR: 5.06), la invasión de la muscular propia (T2; OR: 5,6), la invasión venosa (OR: $5,92)$, la invasión linfática (OR: 4,51) y la presencia de rasgos atípicos que incluyen una depresión central, una erosión o una franca ulceración (OR: 3,00). Los autores concluyen que los mencionados factores deben considerarse en la toma de decisiones, junto al estudio por imágenes y el grado histopatológico.

La Resonancia Magnética (RM) de pelvis y la ultrasonografía endorrectal (USER) son exámenes complementarios en el estudio de un lesión rectal. Se considera que la RM tiene mayor sensibilidad para establecer el compromiso de los ganglios, mientras que la USER puede diferenciar mejor una lesión $\mathrm{T} 1$ de una $\mathrm{T} 2^{14}$. En los últimos años se ha agregado la ultrasonografía endoscópica, que permite estimar la profundidad de una lesión polipoídea rectal y el riesgo de metástasis linfáticas previo a la resección endoscópica con buena sensibilidad ${ }^{15}$. Esta técnica puede cambiar la decisión terapéutica favoreciendo una resección submucosa como alternativa a la mucosectomía tradicional en lesiones de tamaño intermedio ${ }^{16}$.

Actualmente, el PET-CT con 68 Ga-Dotatate, se considera la imagen de elección para el diagnóstico, etapificación y seguimiento de los NETs ${ }^{17}$, técnica que permite mostrar en una imagen de fusión la sobreexpresión local de los receptores de somatostatina y está disponible en los últimos años en nuestro medio ${ }^{18}$.

En nuestra serie los NETs ubicados en el colon, habitualmente, se presentaron como grandes masas tumorales y de acuerdo a una revisión sistemática tienen el peor pronóstico de los TNEs gastro-en- 
tero-hepáticos ${ }^{19}$. Las lesiones ubicadas en el recto, habitualmente son diagnosticadas más precozmente como pólipos o lesiones submucosas pequeñas en colonoscopías efectuadas con fines de screening. La mayoría de las lesiones rectales son menores de $1 \mathrm{~cm}$, están confinadas a la submucosa $(\mathrm{T} 1,89 \%)$ y pueden ser resecadas por vía endoscópica, idealmente con una endosonografía endoscópica para conocer la profundidad del compromiso en la pared intestinal $^{20}$. Sin duda, el manejo de las lesiones elevadas ha cambiado en la última década y el manejo endoscópico se ha sofisticado en forma progresiva, agregándose a la resección endoscópica habitual la disección submucosa endoscópica, la cual muestra progresivamente logros en resecciones comple$\operatorname{tas}^{16,17}$. Si hay elementos de riesgo, debe evaluarse la cirugía radical en pacientes con un riesgo quirúrgico adecuado. En general, los TENs mayores de $2 \mathrm{~cm}$ habitualmente invaden la muscular propia (T2, 5\%) o más allá (T3-T4, 6\%) con un riesgo de alrededor del $60 \%$ de compromiso de los ganglios cercanos, por lo que son candidatos a una resección quirúrgica formal. El riesgo de metástasis ganglionares en las lesiones entre 1 y $2 \mathrm{~cm}$ es de $30 \%{ }^{9}$, motivo por el cual requieren una evaluación caso a caso, considerando la edad y las condiciones comórbidas del paciente. En cuanto al grado, la mayoría de los NETs grado 1 son tributarias de una resección endoscópica, mientras que los grado 2 y 3 requieren de una resección radical si no existe una contraindicación formal. El estudio de imágenes incluye la RM de pelvis que está indicada en lesiones rectales mayores de $2 \mathrm{~cm}$, con invasión de la muscularis propia o más allá (T2-T4) o sospecha de compromiso ganglionar. También es conveniente evaluar con RM de pelvis los casos en que se plantea una cirugía radical luego de una resección endoscópica incompleta (caso 1). El PET-CT con 68 Galio con receptores de somatostatina (DOTATATE) tiene una alta sensibilidad para detectar lesiones NETs, excepto en lesiones muy indiferenciadas o de grado 3 que son poco frecuentes $^{17}$. La CT es de gran relevancia en el estudio de masas tumorales o de enfermedad metastásica, como ocurrió en la mayoría de nuestros casos. Las lesiones avanzadas (T2-T4, N+) tienen indicación de cirugía radical por vía laparoscópica o abierta y una eventual terapia adyuvante según el grado y el estadio de la enfermedad.

Las limitaciones de este estudio son múltiples. Por una parte, los pacientes tienen un estudio de imágenes, histopatológico y la evaluación pronóstica muy heterogéneos, lo que determina tratamientos muy disímiles a lo largo del período estudiado. Los estudios de IHQ fueron variables en el período de 15 años analizado y el $\mathrm{Ki}-67$ y el recuento de mitosis por 10 CAM se investigó sólo en parte de la muestra, lo que refleja la falta de una sistematización en el estudio y manejo de estas lesiones, lo que se ha estandarizado recién en los últimos 5 años ${ }^{14}$. Por otra parte, actualmente el estudio con imágenes se ha desarrollado en forma vertiginosa, incorporándose nuevas tecnologías previamente no disponibles.

De acuerdo con lo aprendido de esta experiencia y la revisión del tema, podemos decir que la evaluación histológica de los NETs debe incluir el grado histológico según el IM y el índice Ki-67 con al menos dos tinciones de IHQ (sinaptofisina y cromogranina A), información que se complementa con la estadificación de acuerdo con el TNM. Sin embargo, es importante determinar la invasión linfática y vascular, variables que no están consideradas en la clasificación TNM y que son consideradas por algunos autores como relevantes en el pronóstico y el riesgo de metástasis linfáticas ${ }^{9,13}$.

\section{Responsabilidades éticas}

Protección de personas y animales. Los autores declaran que para esta investigación no se han realizado experimentos en seres humanos ni en animales.

Confidencialidad de los datos. Los autores declaran que han seguido los protocolos de su centro de trabajo sobre la publicación de datos de pacientes.

Derecho a la privacidad y consentimiento informado. Los autores han obtenido el consentimiento informado de los pacientes y/o sujetos referidos en el articulo. Este documento obra en poder del autor de correspondencia.

\section{Financiación} ción.

El presente trabajo no posee fuente de financia-

\section{Conflicto de intereses}

Los autores declaran no tener ningún conflicto de intereses. 


\section{Bibliografía}

1. Modlin IM, Lye KD, Kidd M. A 5-decade analysis of 13,715 carcinoid tumors. Cancer 2003; 97:934-59.

2. Yao JC, Hassan M, Phan A Dagohoy C, Leary C, Mares J, et al. One hundred years after "carcinoid": Epidemiology o and prognosis factors for neuroendocrine tumors in 35,825 cases in the United States. J Clin Oncol 2008;26:3063-72.

3. García-Carbonero R, Capdevila J, Crespo-Herrero G, Díaz-Pérez JA, Martínez del Prado MP, Alonso Orduña $\mathrm{V}$, et al. Incidence, patterns of care and prognostic factors for outcome of gastroenteropancreatic neuroendocrine tumor (GEP-NETs): results from National Cancer Registry of Spain (RGETNE). Ann Oncol 2010;21:1794-803.

4. Bosman FT, Carneiro F, Hruban RH, Theise ND. WHO Classification of Tumours of the Digestive System, 4th edition. Lyon: International Agency for Research on Cancer, 2010.

5. Ramage JK, De Herder WW, Delle Fave G, Ferolla P, Ferone D, Ito T, et al. ENETS Consensus Guidelines Update for Colorectal Neuroendocrine Neoplasms. Neuroendocrinology 2016;103:139-43.

6. Anthony LB, Strosberg JR, Klimstra DS, Maples WJ, O'Dorisio TM, Warner RR, et al. The NANETS consensus guidelines for the diagnosis and management of gastrointestinal neuroendocrine tumors (NETs): well-differentiated NETs of the distal colon and rectum. Pancreas 2010;39:767-74.

7. Marín A, Vergara JI, Espíndola L,
Sandoval R. Tumor neuroendocrino de células pequeñas del anorrecto. Rev Chil Cir. 2003;55:513-7.

8. Conte B, George B, Overman M, Estrella J, Jiang Z-Q, Sarshekeh AM, et al. High-grade neuroendocrine colorectal carcinomas: a retrospective study of 100 patients. Clinical Colorectal Cancer 2015;15:e1-7.

9. Kojima M, Ikeda K, Saito N, Sakuyama N, Koushi K, Kawano S, et al. Neuroendocrine tumors of the large intestine: Clinicopathological features and predictive factors of lymph node metastasis. Front Oncol. 2016;6:173.

10. Özaslan E, Bayram F, Karaca H, Gürsoy S, Özturk F, Sözuer E, et al. Best prognostic factor of neuroendocrine tumors: Grade or stage? A multidisciplinary single-center study. Turk J Gastroenterol. 2016;27:509-14.

11. Weinstock B, Ward SC, Harpaz N, Warner RRP, Itzkowitz S, Kim MK. Clinical and prognostic features of rectal neuroendocrine tumors. Neuroendocrinology 2013;98:180-7.

12. Shen C, Yin Y, Chen H, Tang, Yin X, Zhou Z, et al. Neuroendocrine tumors of colon and rectum: validation of clinical and prognostic values of the WHO 2010 grading classifications and ENETS. 2016. DOI 10.18632/Oncotarget.13641.

13. Zhou X, Xie H, Xie L, Li J, Fu W. Factors associated with lymph node metastasis in radically resected rectal carcinoids: a systematic review and meta-analysis. J Gastrointest Surg. 2013;17:1689-97.

14. Basuroy R, Haji A, Ramage JK, Quaglia
A, Srirajaskanthan R. Review article: the investigation and management of rectal neuroendocrine tumours. Aliment Pharmacol Ther. 2016;44:332-45.

15. Ishii N, Horiki N, Itoh $\mathrm{T}$, Maruyama M, Matsuda M, Setoyama T, et al. Endoscopic submucosal dissection and preoperative assessment with endoscopic ultrasonography for the treatment of rectal carcinoid tumors. Surg Endosc. 2010;24:1413-9.

16. Wang X, Xiang L, Li A, Han Z, Li Y, Wang Z, et al. Endoscopic submucosal dissection for the treatment of rectal carcinoid tumors $7-16 \mathrm{~mm}$ in diameter. Int J Colorectal Dis. 2015;30:375-80.

17. Moradi F, Jamali M, Barkhodari A, Schneider B, Chin F, Quon A, et al. Spectrum of 68 Ga-DOTA TATE uptake in patients with neuroendocrine tumors. Clin Nuclear Med. 2016;41:e281-7.

18. Amaral H, Pruzzo R, Redondo F, Gil MC, Pizarro A, De la Fuente H, y cols. Una nueva modalidad diagnóstica para la detección de tumores neuroendocrinos con 68Ga-DOTATATE PET/CT. Caso clínico. Rev Med Chile 2009;137:537-41.

19. Mandair D, Caplin ME. Colonic and rectal NET's. Best Pract Res Clin Gastroenterol 2012;26:775-89.

20. McDermott FD, Heeney A, Courtney D, Mohan H, Winter D. Rectal carcinoids: a systematic review. Surg Endosc. 2014;28:2020-6.

21. Bodei L, Sundin A, Kidd M, Prasad V, Modlin IM. The status of neuroendocrine tumor imaging: from darkness to light? Neuroendocrinology 2015;101:1-17. 